\title{
Synthetic biology as an emerging science for biotechnological applications.
}

\author{
Ruann Janser Soares De Castro* \\ Department of Food Science, School of Food Engineering, University of Campinas, 80 Rua Monteiro Lobato, 13083- \\ 862 Campinas, SP, Brazil
}

\section{Editorial}

The combination of important areas such as chemistry, biology, computer sciences, engineering, mathematics and physics originated the concept of biotechnology. Furthermore, the formation of a derivative new field, genetic engineering, elevated biotechnology to the limelight of science in society. Recently, the concept of synthetic biology appeared in order to express the application of biotechnology advances in practical and innovative technologies [1-3].

Thus, synthetic biology can be defined as a tool used to design novel biological pathways, organisms and devices that do not occur naturally or to redesign the existing natural biological systems [1]. This technology enables original solutions in a wide variety of fields such as the discovery of new drugs, fine chemicals, renewable biofuels, vaccines, value-added products, protein therapeutics and cellular reprogramming [1,2].

For being considered a multidisciplinary area, synthetic biology presents numerous scientific and technological opportunities in food, medicine, agriculture, bioremediation, and energy [4].

The production of food ingredients using engineered microorganisms derived from synthetic biology confirms the feasibility of this technique to the food industry. The resveratrol biosynthesis by a genetically modified Saccharomyces cerevisiae [5], the use of Corynebacterium glutamicum as a potential model organism for amino acid production [6-8] and the application of non-conventional yeasts as Hansenula polymorpha, Kluyveromyces lactis, Pichia pastoris and Yarrowia lipolytica in the manufacture of some important bioproducts (biorenewable chemicals, food additives, therapeutic proteins and vaccines) are examples of synthetic biology practical applications [9].

With respect to the agriculture and bioremediation fields, the usage of synthetic biology has some interesting targets. Enrichment of soil with genetically modified microorganisms for a specific crop, and the development of genetically modified strains capable of cleaning up soil contaminated with toxic chemicals are potential applications of this technology [10].

Concerning the biofuel field, the difficulty in fully breaking down the lignocellulose in the plant cell walls is the main issue to be solved. Therefore, synthetic biology is being explored to reduce the resistance of cell walls by developing and prospecting specific enzymes to convert non-food biomass into fuel [11].

Perhaps the clinical applications of synthetic biology are the most prominent considering the recent advances in science. The emergence of the clustered regularly interspaced short palindromic repeats (CRISPR)/CRISPR-associated (Cas) systems facilitate the modeling, designing, and building synthetic gene circuits and other biomolecular components and uses them to rewire and reprogram organisms for a variety of purposes [12,13].

Another remarkable example of innovative technology involving synthetic biology and clinical application is the Brazilian Startup GPhantom, founded by the chemist Michele Ferreira da Costa and the medical physician Felipe Wilker. The company develops a material that mimics human tissue, providing breast and arm simulators designed to replicate interactions such as cysts and venous connections within the body. The mimetizer can be used to calibrate ultrasound machines, and during the development of some technique that requires a simulation in the tissue before testing on humans [14].

Synthetic biology has been considered as the most exciting $21^{\text {st }}$ Century interdisciplinary science, with several promising technologies with potential to change the world $[15,16]$. However, synthetic biology also raises ethical issues which require more debates regarding the biosecurity of this technology.

\section{References}

1. You C, Huang R, Wei X, et al. Protein engineering of oxidoreductases utilizing nicotinamide-based coenzymes, with applications in synthetic biology. Syst Synth Biol. 2017; 208-18.

2. Xie M, Haellman V, Fussenegger M. Synthetic biology - Application-oriented cell engineering. Curr Opin Biotechnol. 2016; 139-48.

3. Singh V. Recent advancements in synthetic biology: Current status and challenges. Gene. 2014; 1 - 11.

4. Luo Y, Lee JK, Zhao H. Challenges and opportunities in synthetic biology for chemical engineers. Chem Eng Sci. 2013; 115-19.

5. Li M, Kildegaard KR, Chen Y, et al. De novo production of resveratrol from glucose or ethanol by engineered Saccharomyces cerevisiae. Metab Eng. 2015; 1-11.

6. Freudl R. Beyond amino acids: Use of the Corynebacterium glutamicum cell factory for the secretion of heterologous proteins. J Biotechnol. 2017; 101-9.

7. Jo S, Yoon J, Lee SM, et al. Modular pathway engineering of Corynebacterium glutamicum to improve xylose utilization and succinate production. J Biotechnol. 2017; 69-78.

8. Woo HM, Park JB. Recent progress in development of synthetic biology platforms and metabolic engineering of Corynebacterium glutamicum. J Biotechnol. 2014; 43-51. 
9. Wagner JM, Alper HS. Synthetic biology and molecular genetics in non-conventional yeasts: Current tools and future advances. Fungal Genet Biol. 2016; 126-136.

10. Tucker JB, Zilinskas RA. The promise and perils of synthetic biology. New Atlantis. 2006; 25-45.

11. Lee SK, Chou H, Ham TS, et al. Metabolic engineering of microorganisms for biofuels production: from bugs to synthetic biology to fuels. Curr Opin Biotechnol. 2008; 556-63.

12. Doudna JA, Charpentier E. The new frontier of genome engineering with CRISPR-Cas9. Science. 2014; 1258096$1-1258096-9$.
13. Benner SA, Sismour AM. Synthetic biology. Nat Rev Genet. 2005; 533-43.

14. Tiengo R. Synergy between university and companies creates environment of innovation in health in Ribeirão Preto. Retrieved in 2017.

15. Jagtap UB, Jadhav JP, Bapat VA, et al. Synthetic biology stretching the realms of possibility in wine yeast research. Int J Food Microbiol. 2017; 24-34.

16. Pretorius IS. Synthetic genome engineering forging new frontiers for wine yeast. Crit Rev Biotechnol. 2017; 112-36.

\section{*Correspondence to:}

Ruann Janser Soares de Castro Department of Food Science

School of Food Engineering, University of Campinas 80 Rua Monteiro Lobato, 13083-862 Campinas, SP, Brazil

Tel: +55 1935212175

Fax: +55 1935212153

E-mail: ruannjanser@hotmail.com

Brazil 